Research report

\title{
Quetiapine treatment reverses depressive-like behavior and reduces DNA methyltransferase activity induced by maternal deprivation
}

\author{
Zuleide M. Ignácio ${ }^{\mathrm{a}, \mathrm{f}}$, Gislaine Z. Réus ${ }^{\mathrm{a}, *}$, Helena M. Abelaira ${ }^{\mathrm{a}}$, Amanda L. Maciel ${ }^{\mathrm{a}}$, \\ Airam B. de Moura ${ }^{a}$, Danyela Matos ${ }^{a}$, Júlia P. Demo ${ }^{a}$, Júlia B.I. da Silva ${ }^{a}$, \\ Fernanda F. Gava ${ }^{a, b}$, Samira S. Valvassori ${ }^{\mathrm{a}, \mathrm{b}}$, André F. Carvalhog ${ }^{\mathrm{g}}$, João Quevedo ${ }^{\mathrm{a}, \mathrm{c}, \mathrm{d}, \mathrm{e}}$ \\ a Laboratory of Neurosciences, Graduate Program in Health Sciences, Health Sciences Unit, University of Southern Santa Catarina, Criciúma, SC, Brazil \\ ${ }^{\mathrm{b}}$ Laboratory of Neural Signaling and Psychopharmacology, Graduate Program in Health Sciences, Health Sciences Unit, University of Southern Santa \\ Catarina (UNESC), Criciúma, SC, Brazil \\ ${ }^{c}$ Center for Translational Psychiatry, Department of Psychiatry and Behavioral Sciences, Medical School, The University of Texas Health Science Center at \\ Houston, Houston, TX, USA \\ ${ }^{\mathrm{d}}$ Center of Excellence on Mood Disorders, Department of Psychiatry and Behavioral Sciences, Medical School, The University of Texas Health Science Center \\ at Houston, Houston, TX, USA \\ e Neuroscience Graduate Program, Graduate School of Biomedical Sciences, The University of Texas Health Science Center at Houston, Houston, TX, USA \\ f Laboratory of Physiology, Pharmacology and Psychopathology, Campus Chapecó, Federal University of South Frontier, Chapecó, Santa Catarina, Brazil \\ ${ }^{g}$ Department of Clinical Medicine and Translational Psychiatry Research Group, Faculty of Medicine, Federal University of Ceará, Fortaleza, CE, Brazil
}

\section{A R T I C L E I N F O}

\section{Article history:}

Received 5 November 2016

Received in revised form

22 November 2016

Accepted 25 November 2016

Available online 29 November 2016

\section{Keywords:}

Quetiapine

Epigenetic

Antidepressant

Maternal deprivation

Major depressive disorder

\begin{abstract}
A B S T R A C T
Stress in early life has been appointed as an important phenomenon in the onset of depression and poor response to treatment with classical antidepressants. Furthermore, childhood trauma triggers epigenetic changes, which are associated with the pathophysiology of major depressive disorder (MDD). Treatment with atypical antipsychotics such as quetiapine, exerts therapeutic effect for MDD patients and induces epigenetic changes. This study aimed to analyze the effect of chronic treatment with quetiapine $(20 \mathrm{mg} / \mathrm{kg}$ ) on depressive-like behavior of rats submitted to maternal deprivation (MD), as well as the activity of histone acetylation by the enzymes histone acetyl transferases (HAT) and deacetylases (HDAC) and DNA methylation, through DNA methyltransferase enzyme (DNMT) in the prefrontal cortex (PFC), nucleus accumbens (NAC) and hippocampus. Maternally deprived rats had a depressive-like behavior in the forced swimming test and an increase in the HDAC and DNMT activities in the hippocampus and NAc. Treatment with quetiapine reversed depressive-like behavior and reduced the DNMT activity in the hippocampus. This is the first study to show the antidepressant-like effect of quetiapine in animals subjected to MD and a protective effect by quetiapine in reducing epigenetic changes induced by stress in early life. These results reinforce an important role of quetiapine as therapy for MDD.
\end{abstract}

(c) 2016 Elsevier B.V. All rights reserved.

\section{Introduction}

A wide variety of research shows that the pathophysiology of MDD involves several biological mechanisms both, in the central nervous system and other physiological systems [64]. The low therapeutic responses of classical antidepressants suggest that MDD is an etiologically heterogeneous disorder [21]. Among the various biological and psychosocial factors, stress during childhood history, such as sexual abuse, physical neglect and family violence were cor-

\footnotetext{
* Corresponding author at: Laboratory of Neurosciences, University of Southern Santa Catarina Criciuma, SC, 88806-000, Brazil.

E-mail address: gislainezilli@hotmail.com (G.Z. Réus).
}

related with a higher vulnerability to MDD and anxiety in adulthood $[14,65]$. In addition, childhood trauma has also been associated with poor response to treatment, similarly to treatment-resistant depression (TRD) [41]. Stress in childhood induces changes in the hypothalamic pituitary adrenal (HPA) axis activity [6,22], a classic physiological phenomenon associated to stress [63]. Studies have shown that changes in HPA axis function are found in MDD individuals who have experienced early life stress [1,54].

Despite the changes in the HPA axis functions, which seem inherent or interrelated to other physiological changes, researches have added an intense focus on epigenetic phenomena intricate in the processes that occur along or much later in individuals exposed to stress at the beginning of life. Among other mechanisms, epigenetic includes processes, which are better known and 
more emphasized in the scientific literature, namely: DNA methylation, which mechanism consist in covalent addition of a methyl group at the $5^{\prime}$ position for cytosine residue, through the DNA methyltransferases (DNMT) enzymes, especially when cytosine is followed by a guanine with a phosphate bond, a sequence called cytosine-phosphate-guanine $(\mathrm{CpG})$ island [32]. An increase on methylation of cytosine in $\mathrm{CpG}$ islands is associated with a reduced gene transcription [28]. Another process refers to post-translational modifications of histone proteins; whose main mechanism consists of acetylation of lysine residues. This process reduces the affinity between proteins and DNA, promoting a relaxation of the chromatin structure and also increasing the recruitment, activation and stabilization of the transcriptional machinery [37]. The acetylation levels along of the chromatin are determined by the balance between HAT, which adds acetyl groups, and HDAC, which removes acetyl groups from lysine residues in histone [30]. The histone acetylation level is determined by the balance between HAT and HDAC enzyme activity, and thus determines the level of transcription of associated gene [29]. The resilience, a phenomenon of resistance and ability to cope with adversity throughout life can be seriously impaired when individuals are subjected to traumatic events during critical periods for brain developmental programming and can result in long-lasting detrimental consequences throughout life and adulthood [34]. In fact, recent literature has pointed out that epigenetic changes resulting from stress in childhood are inherent in a repertoire of biological processes involved in depression $[12,34,58]$. In hippocampus of suicide victims, who suffered various forms of abuse and neglect in childhood, studies showed increased methylation of gene promoter region for glucocorticoid receptor (GR) in parallel with a reduction in the GR mRNA levels and GR expression $[31,38]$. These findings translate results obtained from the hippocampus of rat subjected to maternal deprivation, in which the authors also observed increased depressive-like behavior [67]. Other researchers using MD protocols, also observed increased depressive-like behaviors in parallel with increase of HDAC activity in the rat NAc [48]. It is important to note that the increase in DNA methylation and depressive-like behaviors were reversed with HDAC inhibitors [67]. Indeed, the inactive chromatin is associated with methylated DNA [59], which supports the findings about correlation between inhibition of HDAC and reversing of DNA methylation and depressive behavior. Some studies have shown that classical antidepressants, as well as fastacting drugs seem to reverse the epigenetic alterations involved in previous stress in childhood, in animals subjected to MD [48].

Atypical antipsychotic, also has epigenetic effects by reducing the methylation of genes in the central nervous system $[19,20]$, and peripherally [23]. The Food and Drug Administration (FDA) approved quetiapine in 1997 for the treatment of schizophrenia [9]. Several studies have demonstrated the efficacy, safety, and tolerability of quetiapine in the treatment of MDD and bipolar disorders [3,56]. Noteworthy is the fact that TRD patients showed antidepressant responses when treated with quetiapine [13]. Moreover, in animals subjected to chronic stress, quetiapine, unlike fluoxetine a classical antidepressant, reversed depressive-like behavior and improved hippocampal neurogenesis [66]. Quetiapine has serotonergic properties as a $5-\mathrm{HT}_{1 \mathrm{~A}}$ partial agonist, potent $\alpha 1$ and $\alpha 2$-adrenoceptors antagonist, as also is an inhibitor of the norepinephrine transporter, through its $\mathrm{N}$ desalkylquetiapine metabolite $[50,60]$. These aminergic actions are believed to play a role in its antidepressant properties [27] and increase the therapeutic effect of classical antidepressants [10]. Therefore, the study of drugs targeting neuroepigenetic mechanisms could not only provide insights into the understanding of the mechanisms underlying neurobiological causes of the MDD, but should also accelerate the development of novel pharmacological agents which can be most effective in the treatment of MDD.
Therefore, this study was aimed to investigate the effects of quetiapine administration on the depressive-like behavior, as well as on biochemical parameters related to DNA methylation and histone acetylation in the adult rat subjected to MD during early life.

\section{Experimental procedures}

\subsection{Animals}

Pregnant female Wistar rats (age of 3 months, weight of 250-280 g) were obtained from the breeding colony of Universidade do Extremo Sul Catarinense (UNESC, Criciúma, SC, Brazil) and were housed for one week in the presence of males for sexual experience. At the end of 7 days, pregnant rats $(n=10)$ were housed individually with ad libitum access to food and water. All mothers and pups were kept on a $12 \mathrm{~h}$ light/dark cycle (06:00 a.m. to 06:00 p.m.) at a temperature of $23 \pm 1^{\circ} \mathrm{C}$, including the pups subjected to MD. Male and female pups were used during the maternal deprivation period, but only half of male were subjected to deprivation, according to the protocol below and schematic drawing (Fig. 1). We take care to select females with approximately the same number of animals, so that the number of animals in the separation of the litter was not much different between them. The whole litter, and the mother rat were housed in a clean box, only after the last day of the MD protocol. After this, the animals were weaned in the 21 th postnatal day and housed with five animals per cage. Females were donated to the UNESC vivarium for other studies. Only male rats were used in this research and were primarily divided into two experimental groups: (1) control, non-deprived $(n=23)$, which received no treatment whatsoever; (2) deprived $(n=21)$, which were submitted to MD as described. All experimental procedures involving animals were performed in accordance with the NIH Guide for the Care and Use of Laboratory Animals and the Brazilian Society for Neuroscience and Behavior (SBNeC) recommendations for animal care and with approval by local Ethics Committee under protocol number 82/2012.

\subsection{Maternal deprivation (MD) protocol}

The pups were deprived of the mother for $3 \mathrm{~h}$ per day during the first 10 postnatal days (see Fig. 1). In the first postnatal day, the MD protocol was applied to $50 \%$ of the male pups during 1-10 postnatal days; other males were used as control. The male pups were randomly selected and after the first withdrawal protocol, deprived pups were marked for the deprivation protocols in the following days. The maternal deprivation protocol consisted of removing the pups from the next box. Non-deprived animals remain undisturbed in a home cage with their mother.

\subsection{Drugs and experimental design}

Quetiapine (Seroquel ${ }^{\circledR}$ ) was provided from AstraZeneca (São Paulo, Brazil). Animals received daily intraperitoneal injections of quetiapine $(20 \mathrm{mg} / \mathrm{kg}$ ) for 14 days. The concentration and treatment period considered a first study published by Ignácio et al. [26] that used quetiapine at the doses of 20,40 and $80 \mathrm{mg} / \mathrm{kg}$. Lower concentrations of quetiapine elevated the activity of mitochondrial respiratory chain complexes [26]. Furthermore, an unpublished pilot study from our group showed that the concentration of quetiapine at the dose of $80 \mathrm{mg} / \mathrm{kg}$ administered acutely revealed a sedative effect, while the concentrations of 20 and $40 \mathrm{mg} / \mathrm{kg}$ did not promote sedation, compared to control animals treated with saline. Thus, in the present study we used quetiapine at the dose of $20 \mathrm{mg} / \mathrm{kg}$. Quetiapine was dissolved in saline $(0.9 \%$, $\mathrm{NaCl}$ ) solution (vehicle). Control animals received saline $(0.9 \%$ $\mathrm{NaCl} ; 1.0 \mathrm{ml} / \mathrm{kg}$ ). When the Wistar rats non-deprived and deprived 


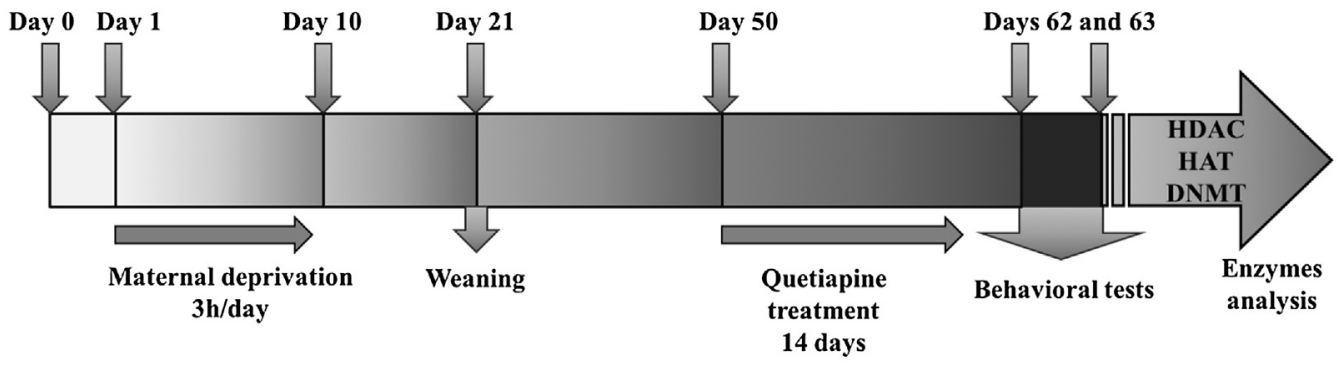

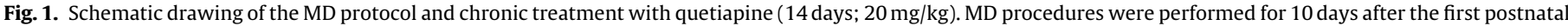

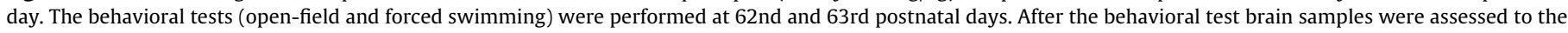
biochemical analysis.

reached 50th postnatal day, the drug treatment was initiated (see Fig. 1). The animals were divided into four groups: (1) nondeprived + saline $(n=11)$; (2) non-deprived + quetiapine $(n=12)$; (3) deprived + saline $(n=10)$; and (4) deprived + quetiapine $(n=11)$.

\subsection{Behavioral tests}

All behavioral tests were conducted in the morning (8:00 a.m.-12:00 p.m.), 60 min after drug treatment, according to the protocols described in the methods section. All behavioral tests were performed by an observer blind to the experimental groups.

\subsubsection{Open field test}

On the 62nd postnatal day (Fig. 1), and 60 min after administration of quetiapine or saline, the animals were subjected to the open-field apparatus, in order to assess possible effects of drug treatment on spontaneous locomotor activity. Analysis of the rat's spontaneous activity was carried out in an open field apparatus, which is an arena $45 \times 60 \mathrm{~cm}$ surrounded by $50 \mathrm{~cm}$ high walls made of brown plywood with a front-facing glass wall. The floor of the open field was divided into 9 rectangles $(15 \times 20 \mathrm{~cm}$ each $)$ by black lines. Animals were gently placed on the left rear rectangle and left to explore the arena for $5 \mathrm{~min}$. The numbers of horizontal (crossing) and vertical (rearing) activities performed by each rat during the 5 min observation period were counted by an experienced observer.

\subsubsection{Forced swimming test}

The forced swimming test was conducted according to the previous reports $[42,45]$. The test involves two individual exposures to a cylindrical tank filled with water in which the rats cannot touch the bottom of the tank or escape. The tank is made of transparent Plexiglas, $80 \mathrm{~cm}$ tall, $30 \mathrm{~cm}$ in diameter, and filled with water $\left(22-23^{\circ} \mathrm{C}\right)$ to a depth of $40 \mathrm{~cm}$. On the 62th postnatal day (Fig. 1), $60 \mathrm{~min}$ after the quetiapine or saline treatment, and after the locomotor activity test in the open field, the rats were individually placed in the cylinder containing water for $15 \mathrm{~min}$ (pre-test session). On the 63rd postnatal day (Fig. 1), the rats received their treatment, and after $60 \mathrm{~min}$ were again subjected to the forced swimming test for a session of $5 \mathrm{~min}$ (test session). The immobility time of the rats was recorded in seconds by an expert observer (the researcher was blind to the experimental groups). After the behavioral tests, all rats were killed by decapitation and the brains were immediately removed. The PFC, hippocampus and NAc were quickly and manually isolated by a skilled researcher, using a magnifying glass, a spatula and a thin brush. The dissection was based on the histological distinctions described by Paxinos and Watson [43]. After the removal of the structures, they were placed in Eppendorf tube and stored in a freezer at $-80^{\circ} \mathrm{C}$ for posterior biochemical analysis. The brain tissues were used for analysis of histone acetylation and DNA methylation levels by observing the enzymatic activity of HAT, HDAC DNMT.

\subsection{Histone acetylatase, histone desacetylase and DNA methyltransferase activities}

\subsubsection{Nuclear extraction and cytosolic fraction separation}

The PFC, hippocampus and NAc were rapidly frozen and stored at $-80 \circ \mathrm{C}$ until nuclear proteins were extracted. The tissue samples, PFC, hippocampus and NAc, frozen at $-80^{\circ} \mathrm{C}$, were subjected to a nuclear extraction protocol from a Nuclear Extraction kit (Sigma, St Louis, USA). Briefly, samples were homogenized in cytoplasmic lysis buffer containing dithiothreitol (DTT) and protease inhibitors. The suspension was kept on ice for 15 min and then centrifuged at $250 \mathrm{xg}$ for $5 \mathrm{~min}$ at $4{ }^{\circ} \mathrm{C}$. The supernatant containing the cytosolic fraction was discarded, and the pellet was resuspended in two volumes of cold cytoplasmic lysis buffer. The suspension was homogenized using a syringe with a small gauge needle and centrifuged at $8000 \mathrm{xg}$ for $20 \mathrm{~min}$ at $4{ }^{\circ} \mathrm{C}$. The pellet was resuspended in an extraction buffer containing DTT and protease inhibitors. The resulting sample was kept in slow agitation for 30-60 min in an orbital shaker at $4{ }^{\circ} \mathrm{C}$. After, the nuclear suspension was centrifuged at $16,000 \mathrm{xg}$ for $5 \mathrm{~min}$ at $4{ }^{\circ} \mathrm{C}$ and the supernatant containing the nuclear extract was transferred to another tube and stored at $-80^{\circ} \mathrm{C}$ until further analysis.

Nuclear extracts from the PFC, hippocampus and NAc were subjected to the HDAC, HAT and DNMT enzymatic activity assay $(\mathrm{n}=5$ for each brain region and enzyme) with the use of HDAC, HAT and DNMT Assay kits (Colorimetric Detection) according to the manufacturer's instructions. Briefly, nuclear extract samples were mixed with HDAC (EPIGENTEK; Base catalog \#P-4034), HAT (EPIGENTEK; Base catalog \#P-4003) or DNMT (EPIGENTEK; Base catalog \#P3009) assay buffer, more assay substrate of the same enzymes, in a 96 well plate and incubated at $30^{\circ} \mathrm{C}$ for $45 \mathrm{~min}$. Concomitantly, a standard curve was performed with serial dilutions of HDAC, HAT or DNMT substrates, as also positive and negative controls were added to the plate. Afterwards, the developer solution was added to the wells, and the plate was incubated at room temperature for $15 \mathrm{~min}$. A colorimetric reading was performed on a fluorescence plate reader with $450 \mathrm{~nm}$ for HDAC, HAT and DNMT activities.

\subsection{Statistical analysis}

All data are presented as mean \pm S.E.M. (standard error of the mean). Differences among experimental groups in the assessment of behavioral tests and HDAC, HAT DNMT enzyme activities were determined by one-way ANOVA, followed by Tukey post hoc test when ANOVA was significant; $\mathrm{p}<0.05$ was considered to be statistically significant. All analyses were carried out in an IBM PC-compatible computer using SPSS. 


\section{Results}

\subsection{Effect of MD and treatment with quetiapine on behavior in the forced swimming and locomotor activity tests}

As represented in Fig. 2a, MD induced depressive-like behavior in adult life, which may be observed by the animal immobility time in the forced swimming test. The depressive-like effect in the animals was significantly reduced in rats chronically treated with quetiapine, as measured by immobility time (Fig. $2 \mathrm{a} ; \mathrm{F}_{(1-41)}=9.047$, $\mathrm{p}<0.001$ ). Fig. 2b shows the effects of MD and quetiapine on the locomotor activity evaluated in the open-field test. Locomotor activity was not different between MD and control animals (nonMD), as measured by number of crossings and rearings. However, quetiapine reduced locomotor activity in MD and control animals (non-MD), but only when compared to saline-treated MD animals, as measured by crossings (Fig. $2 \mathrm{~b} ; \mathrm{F}_{(3-40)}=3.72 ; \mathrm{p}<0.05$ ) and rearings (Fig. 2b; $F_{(3-40)}=5.89 ; p<0.01$ ).

\subsection{Measurement of epigenetic parameters}

\subsubsection{Histone deacetylases (HDAC) activity}

Fig. 3a illustrates the influence of MD and chronic quetiapine treatment on the HDAC activity. The statistical analysis revealed that adulthood MD rats displayed an increase in the HDAC activity in the hippocampus (Fig. 3a; $\mathrm{F}_{(3-16)}=11.11$; $\mathrm{p}<0.001$ ) and NAC (Fig. 3a; $\left.F_{(3-16)}=13.81 ; p<0.001\right)$, but it did not change in PFC (Fig. 3a; $\mathrm{F}_{(3-16)}=1.61 ; \mathrm{p}=0.227$ ). However, chronic treatment with quetiapine did not reduce significantly the increase of HDAC activity in MD animals.

\subsubsection{Histone acetyltransferases (HAT) activity}

As depicted in Fig. 3b, the stress caused by MD did not induce alteration of HAT activity in adult animals. Similarly, chronic treatment with quetiapine did not cause any change in the HAT activity, whether in relation to MD animals treated with saline or control animals (non-MD). Statistical analysis revealed no significant changes in any of brain areas: Hippocampus (Fig. 3b; $\mathrm{F}_{(3-16)}=0.22$; $\mathrm{p}=0.88$ ), NAc (Fig. 3b; $\mathrm{F}_{(3-16)}=2.05 ; \mathrm{p}=0.15$ ), and PFC (Fig. $3 \mathrm{~b}$; $\left.\mathrm{F}_{(3-16)}=0.58 ; \mathrm{p}=0.63\right)$.

\subsubsection{DNA methyltransferases (DNMT) activity}

Fig. $3 c$ shows the influence of MD and chronic quetiapine treatment on the DNMT activity. The statistical analysis revealed that adulthood MD rats displayed an increase of DNMT activity in the hippocampus. MD animals treated with quetiapine also showed an increase in the hippocampal DNMT activity, when compared to animals without stress (non-MD). The quetiapine significantly reduced DNMT activity when compared to MD animals treated with saline, indicating that quetiapine has a pharmacological profile in order to reverse the hippocampal DNA methylation in stressed animals (Fig. 3c; $\left.\mathrm{F}_{(3-16)}=36.56 ; \mathrm{p}<0.001\right)$. In the NAc, the DNMT activity increased significantly in MD animals, both treated with saline and quetiapine (Fig. 3c; $F_{(3-16)}=13.92 ; p<0.001$ ). The chronic treatment with quetiapine did not reduce significantly the increase of DNMT activity in the NAc of MD animals. In the PFC, only MD animals treated with quetiapine had an increase in the DNMT activity compared to animals not stressed (non-MD) and treated with saline (Fig. 3c; $\mathrm{F}_{(3-16)}=3.62 ; \mathrm{p}<0.05$ ). The other groups showed no significant changes in enzyme activity in the PFC.

\section{Discussion}

\subsection{Effect of $M D$ and treatment with quetiapine on behavior in the forced swimming and locomotor activity tests}

This is the first study investigating quetiapine effects in the reduction of depressive-like behavior in adult animals, which were maternally deprived in the first postnatal days. In addition, it was the first study to investigate the effect of quetiapine on epigenetic changes induced by stress in early life. Similar to other studies, the MD increased immobility time in the forced swimming test, indicating a depressive-like behavior in animals, and confirming that the traumatic experiences in early life can culminate in depression in adulthood [49]. Chronic treatment with quetiapine significantly reduced depressive-like behavior. The antidepressant-like effects of quetiapine were also demonstrated in other studies in which adult animals were subjected to chronic stress [66,17]. Additionally, quetiapine reversed the reduction of GABA levels in the hippocampus as well as it reduced the concentrations of corticosterone in the serum of animals subjected to stress [17]. In the studies by Wang et al. [66], the authors observed that quetiapine exercised antidepressant-like action in rodent resistant to treatment with fluoxetine. In the present study, quetiapine induced a significant effect in animals subjected to stress in early life, a condition that has been highlighted as a pivot of the poor response to classical antidepressant treatments in MDD patients [41,68], and in animals displaying depressive-like behaviors [70]. It was observed that animals subjected to MD exhibited depressive-like behavior in adulthood, and escitalopram, a selective serotonin reuptake inhibitor (SSRI), was less effective in reversing the depressive-like behavior [70]. These and other study [17] revealed that quetiapine, as monotherapy and chronically administered exerted significant antidepressant-like effect. In MDD patients, some authors also showed that quetiapine, alone and chronically administered, exercised significant antidepressant effects [35,5], as well as it reduced anxiety in MDD patients with comorbid anxiety [18]. Thus, quetiapine, in addition to function as adjunctive therapy with other antidepressant drugs, also exerts antidepressant effect when administered as monotherapy.

In locomotor activity test, quetiapine reduced crossings and rearing activities, when compared with deprived animals and saline-treated, a result that suggest a sedative function of dose administered $60 \mathrm{~min}$ before testing. Indeed, quetiapine has quick sedative effect after its administration in MDD patients [61], being one of its limitations as therapy for MDD [8]. However, the effect on locomotor activity should be interpreted with some caution, given that in our pilot study, only the concentration of $80 \mathrm{mg} / \mathrm{kg}$ exerted sedative effect, one of the reasons that prompted us to choose the lowest concentration for this study. Some studies have observed that maternally deprived rats, in the 9th and 10th postnatal days, presented an increase in the locomotor activity in adolescence [36] and adult life [47]. In the present study, deprived and saline-treated animals exhibited a slight increase in locomotor activity, when compared to non-deprived animals. Although the increase in activity was not significant, the window between deprived animal's response and small reduction promoted by quetiapine, has the significant effect between quetiapine- and saline-treated deprived animals. It is important to consider that despite of small reduction on locomotor activity, the animals showed a significant reduction in immobility time in the forced swimming test, indicating that a possible small sedative effect of quetiapine did not harm the antidepressant-like effect in animals subjected to stress of maternal deprivation. Notwithstanding, despite of sedative effect, the quetiapine improves the sleep patterns and architecture, even before of the antidepressant effect, and does not seem to exert sedative effect during daytime. In addition, it was suggested that beneficial effect on sleep patterns might be synergistic to other mechanisms elicited 


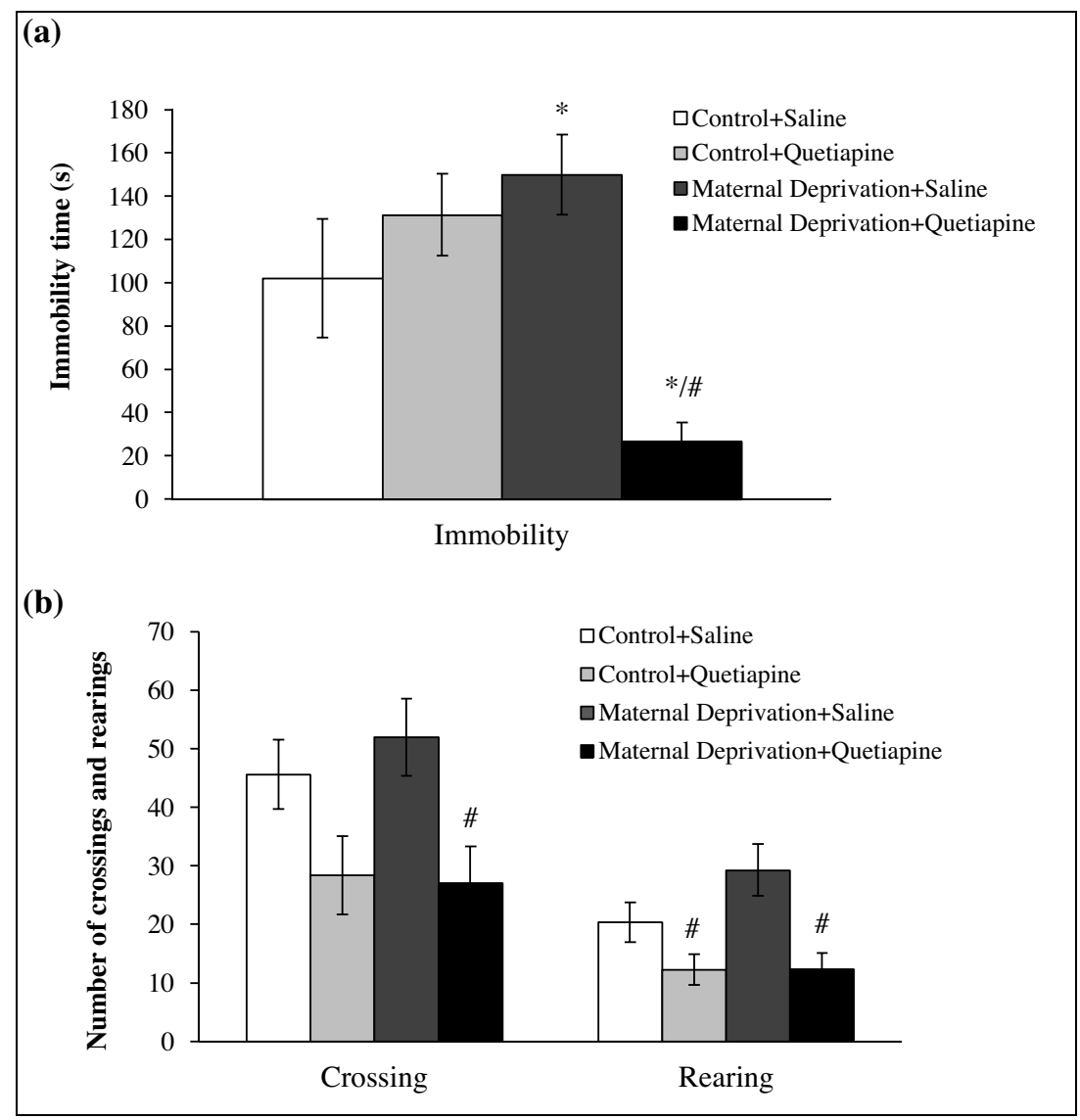

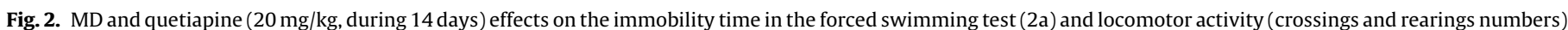

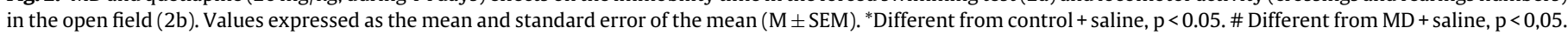

by quetiapine toward the antidepressant therapeutic, when added to classical antidepressants or as monotherapy [61]. Other studies have found that imipramine, a classic antidepressant, reduced immobility in the forced swimming test in diabetic rats, while also reduced locomotor activity in the open field $[7,40]$. Still, regarding the effects of locomotor activity, some studies suggest that reduction of motor activity in the open field is correlated with increased immobility, whereas other authors have shown that locomotor activity is not underlying the performance of animals in the forced swim test [11]. Some authors suggest that other factors may be involved in motor behavior assessed in the open field. For example, in mice, the motor activity was significantly decreased by imipramine chronically administered, after chronic stress for 27 days, while the immobility time also was reduced in the forced swim test. On the other hand, after 21 days of chronic stress, imipramine did not change locomotor activity, compared with the controls or stressed animals [71].

Although quetiapine acts through wide variety of mechanisms, and it is difficult to identify the mechanisms more closely related to the antidepressant effect [51], it is important to note that the drug increases the release of dopamine in brain regions such as PFC [46]. This quetiapine effect is suggested as important to improve affective and cognitive effects underlying depression [46]. Besides dopamine, quetiapine may also has a therapeutic benefit to increase extracellular levels of norepinephrine through blockade of $5-\mathrm{HT}_{2 \mathrm{C}}$ receptors [2], which exert a tonic, inhibitory influence upon dopaminergic and adrenergic pathways in the PFC [39]. Blier and Blondeau [2] also postulated that MDD patients non-responsive to selective serotonin reuptake inhibitors (SSRIs) might be with reduced noradrenergic activity, given that SSRIs reduces firing of noradrenergic neurons in the locus coeruleus, by activating receptors $5-\mathrm{HT}_{2 \mathrm{~A}}$ on inhibitory GABAergic neurons that modulate noradrenergic neurons. Regarding the noradrenergic activity is important to note that quetiapine also has strong blocking action on the norepinephrine transporter and is a potent $\alpha_{2}$-adrenoceptor antagonist $[50,10]$, whose mechanisms increase the synaptic availability of noradrenaline. In addition to the noradrenergic activity, it is also important to consider that increasing dopaminergic activity may be a mechanism related to therapeutic effect on individuals who have poor response to other treatments, as suggested by [24].

\subsection{Effect of MD and treatment with quetiapine on the enzymatic activity of HDAC, HAT and DNMT}

In this work, adult animals, which were maternally deprived, showed an increase in HDAC and DNMT activity in the hippocampus and NAc, confirming other findings described $[67,48]$. Increased activity of these enzymes is associated with lower gene transcription $[28,29]$. Noteworthy is the fact that increasing function of HDACs is associated with depression in animal models and reduced therapeutic response to antidepressants $[15,62]$. Also, it is important to note that chronic treatment with antidepressants can alter the epigenetic changes [48,57], and to mitigate the losses of hippocampal neurogenesis possibly underlying to epigenetic changes resulting from stress in early life [57].

Chronic treatment with quetiapine does not significantly reverse the activity of HDAC in the analyzed brain regions of MD animals, although it has had a tendency to reduce the enzymatic activity in the hippocampus and NAc. However, on the DNMT activity, quetiapine significantly reduced the increase in enzyme activity 


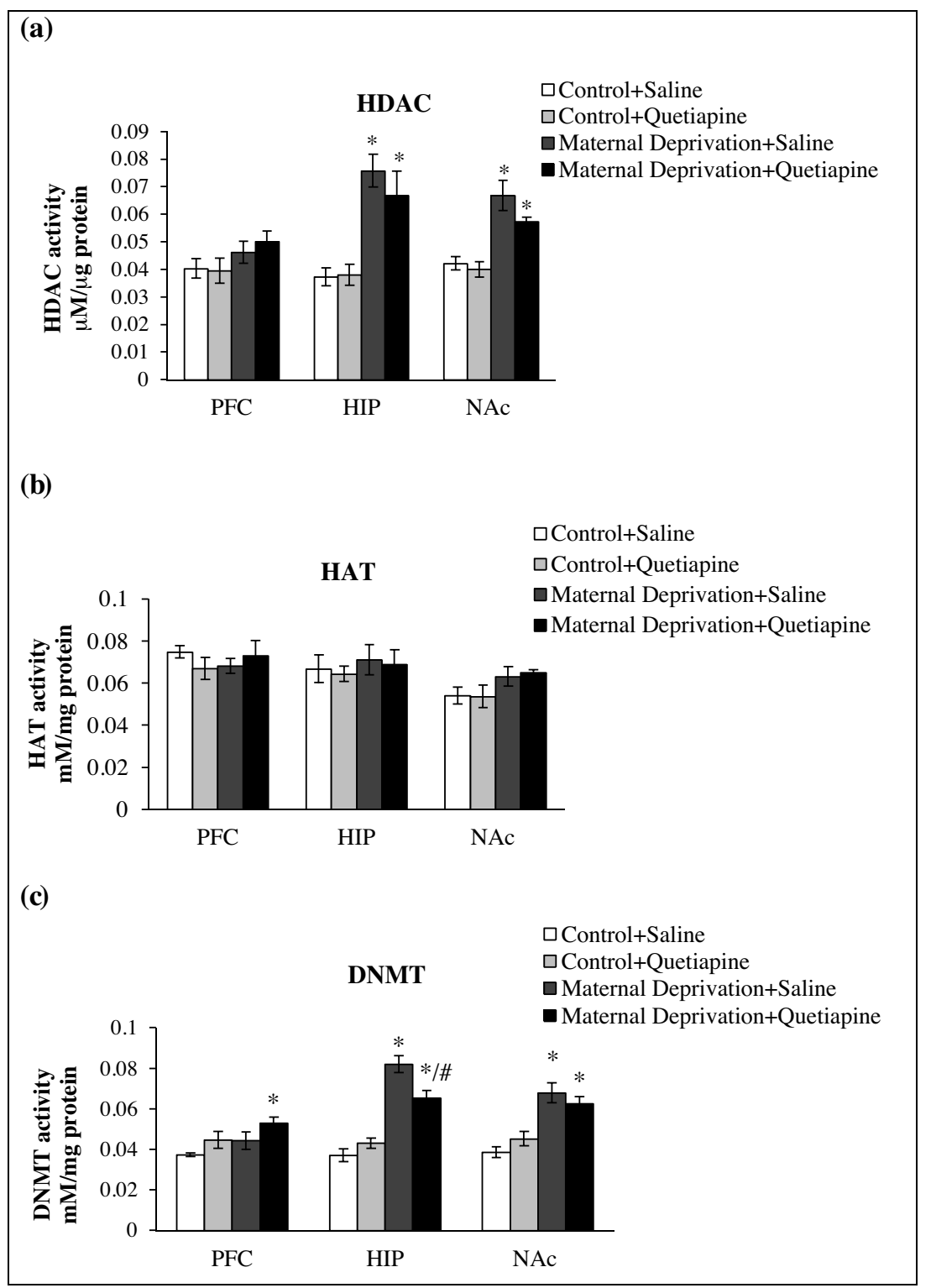

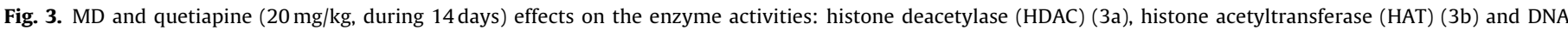

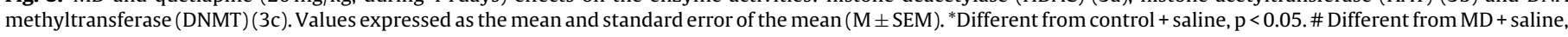
$\mathrm{p}<0,05$.

in the hippocampus and promoted a slight reduction in enzymatic activity in the NAc. Some studies have shown that quetiapine, as well as other dibenzepine derivatives such as clozapine and olanzapine also has epigenetic effect, reducing the methylation of genes in the central nervous system, unlike butyrophenone derivative haloperidol and the piperidyl-benzisoxazole derivative risperidone $[19,20]$. Another important study from human neuroblastoma cell culture showed that quetiapine promotes hypomethylation in wide variety of genes, with the most hypomethylated genes belongs to promoter regions located within CpG island [55]. Another important finding of these last studies was one decreased DNA methylation in the specific $\mathrm{CpG}$ site on the promoter region of the serotonin transporter gene (5-HTT). Genetic polymorphisms in these regions are associated with increased methylation of promoter regions of the 5-HTT gene in lymphoblastic cell lines and with reduced transcriptional activity for the mRNA of serotonin transporter [44]. Moreover, literature reports that the polymorphic variant associated with lower transcriptional activity, also presents greater interaction between gene $\mathrm{x}$ stress in early life, resulting in lifelong depression [25]. Importantly is the fact that genotype that present reduced transcriptional activity also is associated with poor response of patients to treatment with classical antidepressants [53]. Another important evidence is that the polymorphic variant, which showed a higher level of methylation and lower transcriptional activity, was also associated with smaller hippocampal volume in MDD patients [16].

It is also important to highlight that quetiapine reversed the reduction of hippocampal neurogenesis in animals subjected to chronic stress $[33,69]$. In addition, neurogenesis parallel to the antidepressant-like effect promoted by quetiapine, was demonstrated in rats subjected to chronic stress and not responsive to the effect of fluoxetine [66]. The stress of maternal deprivation attenuated differentiation of precursor cells of hippocampal adult neurons and increased DNMT expression. Another interesting finding was that a DNMT inhibitor reversed the reduction by maternal deprivation of neural differentiation of adult neural precursor cells [4]. Thus, the effect of quetiapine on hippocampal neurogenesis may, at 
least in part, be related to its ability to reduce the activity of DNMT enzymes.

The DNMT activity increased in the PFC of MD animals treated with quetiapine. This result seems paradoxical when compared to the results observed in the hippocampus, in this study or results from studies, which observed other brain regions. However, in studies which observed a reduction in DNA methylation in the PFC, the research was directed to specific promoter regions of genes associated with other disorders, where the methylation and demethylation processes appear to require intermediation of other molecular markers [20]. As discussed by the authors, in neurons the promoter methylation is a dynamic process, involving many factors, among which may be the psychopathology and brain regions involved with functions relevant to respective disorder. In addition to lacking data about the function of quetiapine on epigenetic parameters, the scientific literature also lacks data involving the profile of quetiapine and other atypical antipsychotics on DNA methylation mechanisms in the PFC. One study showed that clozapine and risperidone increased methylation of some genes, while in other genes, it appears to show reduced methylation in the PFC, possibly as a positive antipsychotic effect on behaviors related to schizophrenia, or other psychiatric disorders [52]. Therefore, further studies are needed in order to uncover a possible protective or harmful role of quetiapine and other atypical antipsychotics in epigenetic parameters in the PFC.

In conclusion, the results of this study showed that quetiapine exerted antidepressant-like effects in animals subjected to MD stress, an event underlying to MDD. Additionally, ratifying results of the scientific literature, this work data also indicate that previous life stress induces alterations in epigenetic patterns, which can culminate in a reduction in gene transcription process. A most significant finding was the fact that quetiapine induced a significant decrease in DNMT activity in the hippocampus, DNA methylation mechanisms, possibly reversing a hypermethylation induced by stress in childhood.

\section{Acknowledgements}

The Translational Psychiatry Program (USA) is funded by the Department of Psychiatry and Behavioral Sciences, McGovern Medical School, The University of Texas Health Science Center at Houston (UTHealth). Laboratory of Neurosciences (Brazil) is one of the centers of the National Institute for Molecular Medicine (INCT-MM) and one of the members of the Center of Excellence in Applied Neurosciences of Santa Catarina (NENASC). Its research is supported by grants from CNPq (JQ) FAPESC (JQ); Instituto Cérebro e Mente (JQ) and UNESC (JQ and GZR). JQ and AFC are $1 \mathrm{~A} \mathrm{CNPq}$ Research Fellow.

\section{References}

[1] C.V. Baes, C.M. Martins, S.M. Tofoli, M.F. Juruena, Early life stress in depressive patients: HPA axis response to GR and MR agonist, Front. Psychiatry 5 (2014) 2

[2] P. Blier, C. Blondeau, Neurobiological bases and clinical aspects of the use of aripiprazole in treatment-resistant major depressive disorder, J. Affect. Disord. 128 (Suppl. 1) (2011) S3-S10.

[3] B.T. Baune, New developments in the management of major depressive disorder and generalized anxiety disorder: role of quetiapine, Neuropsychiatr. Dis. Treat. 4 (6) (2008) 1181-1191.

[4] S. Boku, H. Toda, S. Nakagawa, A. Kato, T. Inoue, T. Koyama, N. Hiroi, I. Kusumi, Neonatal maternal separation alters the capacity of adult neural precursor cells to differentiate into neurons via methylation of retinoic acid receptor gene promoter, Biol. Psychiatry 77 (4) (2015) 335-344.

[5] B. Bortnick, N. El-Khalili, M. Banov, D. Adson, C. Datto, S. Raines, W. Earley, H. Eriksson, Efficacy and tolerability of extended release quetiapine fumarate (quetiapine XR) monotherapy in major depressive disorder: a placebo-controlled, randomized study, J. Affect. Disord. 128 (1-2) (2011) 83-94.
[6] L.L. Carpenter, J.P. Carvalho, A.R. Tyrka, L.M. Wier, A.F. Mello, M.F. Mello, G.M. Anderson, C.W. Wilkinson, L.H. Price, Decreased adrenocorticotropic hormone and cortisol responses to stress in healthy adults reporting significant childhood maltreatment, Biol. Psychiatry 62 (10) (2007) 1080-1087.

[7] L.B. Ceretta, G.Z. Réus, R.B. Stringari, K.F. Ribeiro, G. Zappellini, B.W. Aguiar, B. Pfaffenseller, C. Lersh, F. Kapczinski, J. Quevedo, Imipramine treatment reverses depressive-like behavior in alloxan-diabetic rats, Diabetes Metab. Res. Rev. 28 (2) (2012) 139-144.

[8] D.S. Cha, R.S. McIntyre, Treatment-emergent adverse events associated with atypical antipsychotics, Expert. Opin. Pharmacother. 13 (11) (2010) 1587-1598.

[9] S.M. Cheer, A.J. Wagstaff, Quetiapine -A review of its use in the management of schizophrenia, CNS Drugs 18 (3) (2004) 173-199.

[10] O. Chernoloz, M. El Mansari, P. Blier, Effects of sustained administration of quetiapine alone and in combination with a serotonin reuptake inhibitor on norepinephrine and serotonin transmission, Neuropsychopharmacology 37 (7) (2012) 1717-1728.

[11] J.N. Crawley, J.K. Belknap, A. Collins, J.C. Crabbe, W. Frankel, N. Henderson, R.J Hitzemann, S.C. Maxson, L.L. Miner, A.J. Silva, J.M. Wehner, A. Wynshaw-Boris, R. Paylor, Behavioral phenotypes of inbred mouse strains: implications and recommendations for molecular studies, Psychopharmacology (Berl.) 132 (2) (1997) 107-124

[12] V.S. Dalton, E. Kolshus, D.M. Mcloughlin, Epigenetics and depression: return of the repressed, J. Affect. Disord. 155 (2014) 1-12.

[13] E.J. Daly, M.H. Trivedi, A review of quetiapine in combination with antidepressant therapy in patients with depression, Neuropsychiatr. Dis. Treat. 3 (6) (2007) 855-867.

[14] N.P. Daskalakis, R.C. Bagot, K.J. Parker, C.H. Vinkers, E.R. de Kloet, The three-hit concept of vulnerability and resilience: toward understanding adaptation to early-life adversity outcome, Psychoneuroendocrinology 38 (9) (2013) $1858-1873$.

[15] M. Dyrvig, H.H. Hansen, S.H. Christiansen, D.P. Woldbye, J.D. Mikkelsen, J. Lichota, Epigenetic regulation of Arc and c-Fos in the hippocampus after acute electroconvulsive stimulation in the rat, Brain Res. Bull. 88 (5) (2012) $507-513$.

[16] M.C. Eker, O. Kitis, H. Okur, O.D. Eker, E. Ozan, S. Isikli, N. Akarsu, A.S. Gonul, Smaller hippocampus volume is associated with short variant of 5-HTTLPR polymorphism in medication-free major depressive disorder patients, Neuropsychobiology 63 (1) (2011) 22-28.

[17] S.M.K.S. El Dine, An increase in GABA content in Hippocampus of Albino rats exposed to chronic restraint model and treated by Quetiapine for 3 weeks, J. Pharm. Innov. 3 (12) (2015) 89-93.

[18] I. Galynker, A. Khan, Y. Grebchenko, A. Ten, L. Malaya, P. Yanowitch, L.J. Cohen, Low-dose risperidone and quetiapine as monotherapy for comorbid anxiety and depression, J. Clin. Psychiatry 66 (4) (2005) 544.

[19] A. Guidotti, J. Auta, Y. Chen, J.M. Davis, E. Dong, D.P. Gavin, D.R. Grayson, F. Matrisciano, G. Pinna, R. Satta, R.P. Sharma, L. Tremolizzo, P. Tueting, Epigenetic GABAergic targets in schizophrenia and bipolar disorder Neuropharmacology 60 (7-8) (2011) 1007-1016.

[20] A. Guidotti, D.R. Grayson, DNA methylation and demethylation as targets for antipsychotic therapy, Dialogues Clin. Neurosci. 16 (3) (2014) 419-429.

[21] G. Hasler, Pathophysiology of depression: do we have any solid evidence of interest to clinicians? World Psychiatry 9 (3) (2010) 155-161.

[22] C. Heim, D.J. Newport, S. Heit, Y.P. Graham, M. Wilcox, R. Bonsall, A.H. Miller, C.B. Nemeroff, Pituitary-adrenal and autonomic responses to stress in women after sexual and physical abuse in childhood, JAMA 284 (5) (2000) 592-597.

[23] L.C. Houtepen, A.H. van Bergen, C.H. Vinkers, M.P. Boks, DNA methylation signatures of mood stabilizers and antipsychotics in bipolar disorder, Epigenomics 8 (2) (2016) 197-208.

[24] M.C. Hsiao, K.J. Lin, C.Y. Liu, D.B. Schatz, The interaction between dopamine transporter function, gender differences, and possible laterality in depression, Psychiatry Res. 211 (1) (2013) 72-77.

[25] Z.M. Ignácio, G.Z. Réus, H.M. Abelaira, J. Quevedo, Epigenetic and epistatic interactions between serotonin transporter and brain-derived neurotrophic factor genetic polymorphism: insights in depression, Neuroscience 275 (2014) 455-468.

[26] Z.M. Ignácio, G.Z. Réus, H.M. Abelaira, S.E. Titus, A.S. Carlessi, J.R. da Luz, B.I. Matias, L. Bruchchen, M. Carvalho-Silva, L.M. Gomes, J. Rebelo, E.L. Streck, J. Quevedo, Acute and chronic treatments with quetiapine increase mitochondrial respiratory chain complex activity in the rat brain, Curr. Neurovasc. Res. 12 (3) (2015) 283-292.

[27] N.H. Jensen, R.M. Rodriguiz, M.G. Caron, W.C. Wetsel, R.B. Rothman, B.L. Roth, $\mathrm{N}$-desalkylquetiapine, a potent norepinephrine reuptake inhibitor and partial 5-HT1A agonist, as a putative mediator of quetiapine's antidepressant activity, Neuropsychopharmacology 33 (10) (2008) 2303-2312.

[28] P. Jones, D. Takai, The role of DNA methylation in mammalian epigenetics, Science 293 (2001) 1068-1070.

[29] T. Kouzarides, Chromatin modifications and their function, Cell 128 (2007) 693-705.

[30] M.H. Kuo, C.D. Allis, Roles of histone acetyltransferases and deacetylases in gene regulation, Bioessays 20 (8) (1998) 615-626.

[31] B. Labonte, V. Yerko, J. Gross, N. Mechawar, M.J. Meaney, M. Szyf, G. Turecki, Differential glucocorticoid receptor exon $1 \mathrm{~B}, 1 \mathrm{C}$, and $1 \mathrm{H}$ expression and methylation in suicide completers with a history of childhood abuse, Biol. Psychiatry 72 (2012) 41-48. 
[32] R. Lister, M. Pelizzola, R.H. Dowen, R.D. Hawkins, G. Hon, J. Tonti-Filippini, J.R. Nery, L. Lee, Z. Ye, Q.M. Ngo, L. Edsall, J. Antosiewicz-Bourget, R. Stewart, V. Ruotti, A.H. Millar, J.A. Thomson, B. Ren, J.R. Ecker, Human DNA methylomes at base resolution show widespread epigenomic differences, Nature 462 (7271) (2009) 315-322.

[33] C. Luo, H. Xu, X.M. Li, Quetiapine reverses the suppression of hippocampal neurogenesis caused by repeated restraint stress, Brain Res. 1063 (1) (2005) 32-39.

[34] S. Maccari, H.J. Krugers, S. Morley-Fletcher, M. Szyf, P.J. Brunton, The consequences of early-life adversity: neurobiological, behavioural and epigenetic adaptations, J. Neuroendocrinol. 26 (10)(2014) 707-723.

[35] N. Maneeton, B. Maneeton, M. Srisurapanont, S.D. Martin, Quetiapine monotherapy in acute phase for major depressive disorder: a meta-analysis of randomized, placebo-controlled trials, BMC Psychiatry 12 (2012) 160.

[36] E.M. Marco, W. Adriani, R. Canese, F. Podo, M.P. Viveros, G. Laviola, Enhancement of endocannabinoid signalling during adolescence: modulation of impulsivity and long-term consequences on metabolic brain parameters in early maternally deprived rats, Pharmacol. Biochem. Behav. 86 (2007) $334-345$.

[37] R. Marmorstein, R.C. Trievel, Histone modifying enzymes: structures, mechanisms, and specificities, Biochim. Biophys. Acta (BBA) Gene Regul. Mech. 1789 (1) (2009) 58-68.

[38] P.O. McGowan, A. Sasak i, A.C. D’Alessio, S. Dymov, B. Labonté, M. Szyf, G. Turecki, M.J. Meaney, Epigenetic regulation of the glucocorticoid receptor in human brain associates with childhood abuse, Nat. Neurosci. 12 (3) (2009) 342-348.

[39] M.J. Millan, A. Gobert, F. Lejeune, A. Dekeyne, A. Newman-Tancredi, V. Pasteau, J.M. Rivet, D. Cussac, The novel melatonin agonist agomelatine (S20098) is an antagonist at 5-hydroxytryptamine2C receptors, blockade of which enhances the activity of frontocortical dopaminergic and adrenergic pathways, J. Pharmacol. Exp. Ther. 306 (2003) 954-964.

[40] R.I. Nadeem, H.I. Ahmed, E.E. El-Denshary, Effect of imipramine, paroxetine, and lithium carbonate on neurobehavioral changes of streptozotocin in rats: impact on glycogen synthase kinase-3 and blood glucose level, Neurochem. Res. 40 (9) (2015) 1810-1818.

[41] V. Nanni, R. Uher, A. Danese, Childhood maltreatment predicts unfavorable course of illness and treatment outcome in depression: a meta analysis, Am. J. Psychiatry 169 (2) (2012) 141-151.

[42] C.F. Ortmann, G.Z. Réus, Z.M. Ignácio, H.M. Abelaira, S.E. Titus, P. de Carvalho, C.O. Arent, M.A. Dos Santos, B.I. Matias, M.M. Martins, A.M. de Campos, F. Petronilho, L.J. Teixeira, M.O. Morais, E.L. Streck, J. Quevedo, F.H. Reginatto, Enriched flavonoid fraction from CecropiapachystachyaTrécul leaves exerts antidepressant-like behavior and protects brain against oxidative stress in rats subjected to chronic mild stress, Neurotox. Res. 29 (4) (2016) 469-483.

[43] G. Paxinos, C. Watson, The Rat Brain: Stereotaxic Coordinates, 2nd ed., Academic San Diego, 1986.

[44] R. Philibert, A. Madan, A. Andersen, R. Cadoret, H. Packer, H. Sandhu, Serotonin transporter mRNA levels are associated with the methylation of an upstream CpG island, Am. J. Med. Genet. B Neuropsychiatr. Genet. 144B (2007) 101-105.

[45] R.D. Porsolt, A. Bertin, M. Jalfre, Behavioural despair in mice: a primary screening test for antidepressants, Arch. Int. Pharmacodyn. Ther. 229 (1977) 327-336.

[46] E. Prieto, J.A. Micó, J.J. Meana, S. Majadas, Neurobiological bases of quetiapineantidepresant effect in the bipolar disorder, Actas Esp. Psiquiatr. 38 (1) (2010) 22-32.

[47] G. Rentesi, K. Antoniou, M. Marselos, M. Syrrou, Z. Papadopoulou-Daifoti, M. Konstandi, Early maternal deprivation-induced modifications in the neurobiological, neurochemical and behavioral profile of adult rats, Behav. Brain Res. 244 (2013) 29-37.

[48] G.Z. Réus, H.M. Abelaira, M.A. dos Santos, A.S. Carlessi, D.B. Tomaz, M.V. Neotti, J.L. Liranço, C. Gubert, M. Barth, F. Kapczinski, J. Quevedo, Ketamine and imipramine in the nucleus accumbens regulate histone deacetylation induced by maternal deprivation and are critical for associated behaviors, Behav. Brain Res. 256 (2013) 451-456.

[49] G.Z. Réus, A.S. Carlessi, S.E. Titus, H.M. Abelaira, Z.M. Ignácio, J.R. da Luz, B.I. Matias, L. Bruchchen, D. Florentino, A. Vieira, F. Petronilho, J. Quevedo, A single dose of S-ketamine induces long-term antidepressant effects and decreases oxidative stress in adulthood rats following maternal deprivation, Dev. Neurobiol. 75 (11) (2015) 1268-1281.

[50] E. Richelson, T. Souder, Binding of antipsychotic drugs to human brain receptors focus on newer generation compounds, Life Sci. 68 (2000) 29-39.
[51] C.F. Saller, A.I. Salama, Seroquel: biochemical profile of a potential atypical antipsychotic, Psychopharmacology (Berl.) 112 (2-3) (1993) 285-292.

[52] M.L. Santoro, V.K. Ota, R.S. Stilhano, P.N. Silva, C.M. Santos, M.C. Diana, A. Gadelha, R.A. Bressan, M.I. Melaragno, S.W. Han, V.C. Abílio, S.I. Belangero, Effect of antipsychotic drugs on gene expression in the prefrontal cortex and nucleus accumbens in the spontaneously hypertensive rat (SHR), Schizophr. Res. 157 (1-3) (2014) 163-168.

[53] A. Serretti, M. Kato, D. De Ronchi, T. Kinoshita, Meta-analysis of serotonin transporter gene promoter polymorphism (5-HTTLPR) association with selective serotonin reuptake inhibitor efficacy in depressed patients, Mol. Psychiatry 12 (2007) 247-257.

[54] N. Strüber, D. Strüber, G. Roth, Impact of early adversity on glucocorticoid regulation and later mental disorders, Neurosci. Biobehav. Rev. 38 (2014) $17-37$.

[55] H. Sugawara, M. Bundo, T. Asai, F. Sunaga, J. Ueda, J. Ishigooka, K. Kasai, T. Kato, K. Iwamoto, Effects of quetiapine on DNA methylation in neuroblastoma cells Prog, Neuropsychopharmacol. Biol. Psychiatry 56 (2015) 117-122.

[56] T. Suppes, E. Vieta, S. Liu, M. Brecher, B. Paulsson, Trial 127 Investigators.,. Maintenance treatment for patients with bipolar I disorder: results from a north american study of quetiapine in combination with lithium or divalproex (trial 127), Am. J. Psychiatry 166 (4) (2009) 476-488.

[57] D. Suri, V. Veenit, A. Sarkar, D. Thiagarajan, A. Kumar, E.J. Nestler, S. Galande, V.A. Vaidya, Early stress evokes age-dependent biphasic changes in hippocampal neurogenesis, BDNF expression, and cognition, Biol. Psychiatry 73 (7) (2013) 658-666.

[58] M. Szyf, The early-life social environment and DNA methylation, Clin. Genet. 81 (2012) 341-349.

[59] M. Tachibana, Y. Matsumura, M. Fukuda, H. Kimura, Y. Shinkai, G9a/GLP complexes independently mediate H3K9 and DNA methylation to silence transcription, EMBO J. 27 (20) (2008) 2681-2690.

[60] Y. Tanibuchi, Y. Fujita, M. Kohno, T. Ishima, Y. Takatsu, M. Iyo, K. Hashimoto Effects of quetiapine on phencyclidine-induced cognitive deficits in mice: a possible role of alpha1-adrenoceptors, Eur. Neuropsychopharmacol. 19 (12) (2009) 861-867.

[61] D. Todder, S. Caliskan, B.T. Baune, Night locomotor activity and quality of sleep in quetiapine-treated patients with depression, J. Clin. Psychopharmacol. 26 (6) (2006) 638-642.

[62] N.M. Tsankova, O. Berton, W. Renthal, A. Kumar, R.L. Neve, E.J. Nestler, Sustained hippocampal chromatin regulation in a mouse model of depression and antidepressant action, Nat. Neurosci. 9 (4) (2006) 519-525.

[63] C. Tsigos, G.P. Chrousos, Hypothalamic-pituitary-adrenal axis, neuroendocrine factors and stress, J. Psychosom. Res. 53 (4) (2002) 865-871.

[64] J. Verduijn, Y. Milaneschi, R.A. Schoevers, A.M. van Hemert, A.T. Beekman, B.W. Penninx, Pathophysiology of major depressive disorder: mechanisms involved in etiology are not associated with clinical progression, Transl. Psychiatry 5 (2015) e649.

[65] A.M. Vranceanu, S.E. Hobfoll, R.J. Johnson, Child multi-type maltreatment and associated depression and PTSD symptoms: the role of social support and stress, Child. Abuse Negl. 31 (1) (2007) 71-84.

[66] Y. Wang, T. Chang, Y.C. Chen, R.G. Zhang, H.N. Wang, W.J. Wu, Z.W. Peng, Q.R. Tan, Quetiapine add-on therapy improves the depressive behaviors and hippocampal neurogenesis in fluoxetine treatment resistant depressive rats, Behav. Brain Res. 253 (2013) 206-211.

[67] I.C. Weaver, N. Cervoni, F.A. Champagne, A.C. D’Alessio, S. Sharma, J.R. Seckl, S. Dymov, M. Szyf, M.J. Meaney, Epigenetic programming by maternal behavior, Nat. Neurosci. 7 (8) (2004) 847-854.

[68] L.M. Williams, C. Debattista, A.M. Duchemin, A.F. Schatzberg, C.B. Nemeroff, Childhood trauma predicts antidepressant response in adults with major depression: data from the randomized international study to predict optimized treatment for depression, Transl. Psychiatry. 6 (2016) e799.

[69] H. Xu, Z. Chen, J. He, S. Haimanot, X. Li, L. Dyck, X.M. Li, Synergetic effects of quetiapine and venlafaxine in preventing the chronic restraint stress-induced decrease in cell proliferation and BDNF expression in rat hippocampus, Hippocampus 16 (6) (2006) 551-559.

[70] Y. Zhang, Y. Wang, L. Wang, M. Bai, X. Zhang, X. Zhu, Dopamine receptor D2 and associated microRNAs are involved in stress susceptibility and resistance to escitalopram treatment, Int. J. Neuropsychopharmacol. 18 (8) (2015) (pii, pyv025).

[71] J. Zhao, Y.H. Jung, C.G. Jang, K.H. Chun, S.W. Kwon, J. Lee, Metabolomic identification of biochemical changes induced by fluoxetine and imipramine in a chronic mild stress mouse model of depression, Sci. Rep. 5 (2015) 8890. 